\title{
Tunneling ionization of deep centers in high-frequency electric fields
}

\author{
S. D. Ganichev, ${ }^{1,2}$ I. N. Yassievich, ${ }^{2}$ V. I. Perel, ${ }^{2}$ H. Ketterl, ${ }^{1}$ and W. Prettl ${ }^{1}$ \\ ${ }^{1}$ Institut für Experimentelle und Angewandte Physik, Universität Regensburg, 93040 Regensburg, Germany \\ ${ }^{2}$ A. F. Ioffe Physico-Technical Institute, Russian Academy of Sciences, 194021 St. Petersburg, Russia \\ (Received 18 May 2001; revised manuscript received 26 October 2001; published 4 February 2002)
}

\begin{abstract}
A theoretical and experimental study of the tunneling ionization of deep impurities in semiconductors has been carried out for high-frequency alternating electric fields. It is shown that tunneling ionization occurs by phonon-assisted tunneling which proceeds at high electric field strengths into direct tunneling without involving phonons. In the quasistatic regime of low frequencies the tunneling probability is independent of frequency. Raising the frequency leads to an enhancement of the tunneling ionization. The transition from the quasistatic limit to frequency-dependent tunneling is determined by the tunneling time which, in the case of impurities interacting with thermal phonons, is controlled by the temperature. In both the quasistatic and high-frequency limits, the application of an external magnetic field perpendicular to the electric field reduces the ionization probability when the cyclotron frequency becomes larger than the reciprocal tunneling time.
\end{abstract}

DOI: $10.1103 /$ PhysRevB.65.085203

PACS number(s): 73.40.Gk, 72.30.+q, 71.55.-i, 03.65.Sq

\section{INTRODUCTION}

One of the main manifestations of quantum mechanics is tunneling. Tunneling in alternating potentials is important in a variety of physical phenomena like field emission, interband breakdown, charge exchange between deep-lying impurity centers in semiconductors, tunneling chemical reactions, Josephson junctions, resonance tunneling, Coulomb blockade, the destruction of adiabatic invariants, and others.

Recently an enhancement of the tunneling probability in the high-frequency electric field of lasers compared to the tunneling in static fields has been observed. ${ }^{1}$ The transition between the quasistatic regime, where tunneling does not depend on the oscillation frequency of the applied radiation electric field, and the high-frequency regime, where the enhancement of tunneling occurs, is determined by the tunneling time. Experimentally deep impurities in semiconductors have been ionized by contactless application of alternating electric fields of a strong far-infrared molecular lasers. The photon energies of the laser fields were much smaller than the impurity binding energies, thus avoiding one-photon ionization. In contrast to the tunneling ionization of atoms, where only electron tunneling takes place, the tunneling ionization of impurities in solids is accomplished by two simultaneous tunneling processes: electron tunneling and the redistribution of the vibrational system by defect tunneling. ${ }^{2}$ At not too low temperatures and not too high electric field strengths such phonon-assisted tunneling is the dominant ionization mechanism whereas at low temperatures or very high fields direct carrier tunneling from the ground state into the continuum controls the ionization process without participation of phonons. ${ }^{3}$

In the case of deep impurities the tunneling time, which determines the transition from the quasistatic to the highfrequency regime, is the time of the redistribution of the defect vibrational system upon ionization by tunneling. ${ }^{4}$ As long as phonons are involved in the ionization process, this time depends strongly on the temperature as well as on the details of the impurity potential structure. Measurements of the ionization rate as a function of the electric field strength allow one to determine tunneling times which are intensively discussed in the literature. ${ }^{5,6}$

Here we present a detailed experimental and theoretical study of the frequency dependence of the tunneling ionization of deep impurities in semiconductors. It is demonstrated that the tunneling probability may be increased by many orders of magnitude if the radiation frequency gets much larger than the inverse tunneling time. This is shown to be due to the absorption of radiation energy by the tunneling carrier under the potential barrier formed by the attractive potential well of the impurity and the external electric field of the radiation. Therefore the carrier leaves the barrier at higher energy, yielding an effectively narrower barrier.

\section{THEORY}

\section{A. Tunneling ionization}

The tunneling ionization of deep impurities will be treated in a semiclassical approximation and in the adiabatic limit for the phonon system. For the sake of simplicity and to be specific we will discuss electrons only though the experimental investigations involve either electrons or holes. Due to electron-phonon interaction, the system, consisting of local impurity vibrations and an electron, is characterized by two adiabatic potentials $U_{1}(x)$ and $U_{2}(x)$ as a function of a configuration coordinate $x$. These adiabatic potentials correspond to the electron bound to the impurity and the electron detached from the impurity with zero kinetic energy, respectively. ${ }^{7,8}$ Two main possible configuration-coordinate diagrams will be considered: (a) for strong electron-phonon coupling where self-trapping occurs as, for example, in the case of DX and EL2 centers in III-V semiconductors and (b) for the case of weak electron-phonon interaction which is realized with substitutional impurities.

In the case when a free electron has kinetic energy $\varepsilon$, the adiabatic potential is $U_{2 \varepsilon}(x)=U_{2}(x)+\varepsilon$. In the presence of an electric field an electron can be emitted at a negative kinetic energy $\varepsilon<0$ due to tunneling through the triangular barrier formed with the binding electron potential well by the 
a)

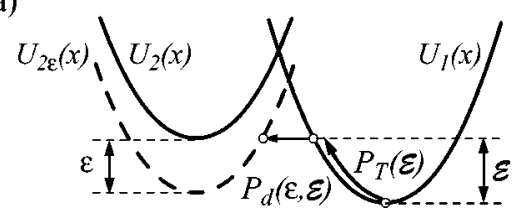

b)

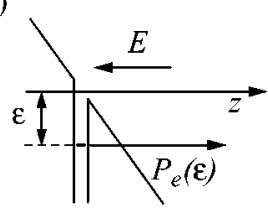

FIG. 1. Illustration of the tunneling ionization of deep centers in a static electric field: (a) thermal excitation and defect tunneling and (b) electron tunneling with probabilities $P_{T}, P_{d}$, and $P_{e}$, respectively.

electric field [see Fig. 1(b)]. In this case the electric-fieldstimulated thermal emission of carriers can be described by a three-step process.

(i) Thermal excitation drives the vibrational system to an energy level $\mathcal{E}$ in the bound-state potential $U_{1}(x)$ [see Fig. 1(a)]. The probability of this process is $P_{T}(\mathcal{E})$ $\propto \exp (-\mathcal{E} / k T)$.

(ii) A tunneling reconstruction of the vibrational system occurs corresponding to a tunneling at energy $\mathcal{E}$ from the bound potential $U_{1}(x)$ to the ionized potential $U_{2 \varepsilon}(x)$ with the probability $P_{d}(\varepsilon, \mathcal{E})$ [see Fig. 1(a)]. This process will be called defect tunneling in contrast to electron tunneling.

(iii) Simultaneously the electron leaves the impurity by tunneling in the electric field at energy $\varepsilon<0$ with the probability $P_{e}(\varepsilon)$ [see Fig. 1(b)]. The electric field acts on electron tunneling only and the stimulation of thermal tunneling ionization of impurities is caused by the lowering of the ionized adiabatic potential from $U_{2}(x)$ to $U_{2 \varepsilon}(x)$ with $\varepsilon$ $<0$ [Fig. 1(a)].

The ionization probability of the total process $P(\varepsilon, \mathcal{E})$ is determined by

$$
P(\varepsilon, \mathcal{E})=P_{T}(\mathcal{E}) P_{d}(\varepsilon, \mathcal{E}) P_{e}(\varepsilon) .
$$

The probability $P_{d}(\varepsilon, \mathcal{E})$ of the tunneling reconstruction of the vibrational system in semiclassical approximation is determined applying the Landau technique of complex semiclassical trajectories. ${ }^{7,9}$ The tunneling trajectories and potential barriers for weak and strong electron-phonon interaction are shown in Fig. 2.

After Landau the trajectories are split into two parts both under barriers formed by the potentials $U_{1}(x)$ and $U_{2 \varepsilon}(x)$ where $x=x_{c \varepsilon}$ is the intersection of the potentials. The system tunnels at energy level $\mathcal{E}$ from the turning point $a_{1}$ to $x_{c \varepsilon}$

a)

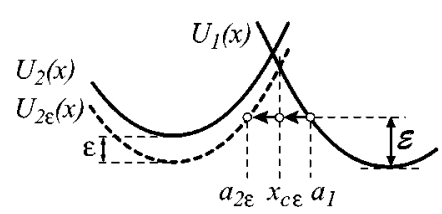

b)

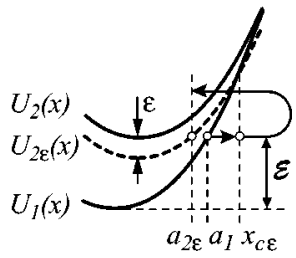

FIG. 2. Blown-up representations of the tunneling trajectories for (a) strong electron-phonon coupling (autolocalization) and (b) weak electron-phonon coupling. The dashed curves show the potential $U_{2 \varepsilon}(x)$ of the system: an ionized impurity and an electron with negative kinetic energy $\varepsilon$ obtained by electron tunneling in an electric field.

under the potential $U_{1}(x)$ and from $x_{c \varepsilon}$ to the turning point $a_{2 \varepsilon}$ under $U_{2 \varepsilon}(x)$. The tunneling probability depends exponentially on the imaginary part of the principal function evaluated along the trajectory linking the turning points $a_{1}$ and $a_{2 \varepsilon}$. Then the probability of defect tunneling is given by

$$
P_{d}(\varepsilon, \mathcal{E}) \propto \exp \left[-2\left(S_{2 \varepsilon}-S_{1 \varepsilon}\right)\right]
$$

where

$$
S_{1 \varepsilon}=\frac{\sqrt{2 M}}{\hbar} \int_{a_{1}}^{x_{c \varepsilon}} \sqrt{U_{1}(x)-\left(\mathcal{E}-\varepsilon_{T}\right)} d x
$$

and

$$
S_{2 \varepsilon}=\frac{\sqrt{2 M}}{\hbar} \int_{a_{2 \varepsilon}}^{x_{c \varepsilon}} \sqrt{U_{2 \varepsilon}(x)-\left(\mathcal{E}-\varepsilon_{T}\right)} d x .
$$

Here $M$ is the effective mass of the impurity complex determined by the vibrational mode, and $\varepsilon_{T}$ is the the thermal ionization energy of the electron determined as a difference between minima of the parabola corresponding to electron detached and electron bound to the impurity, respectively. We use the one-mode approximation for local vibrations. This approximation is justified because the main modulation of electron binding energy is due to the breathing mode of the impurity complex.

The probability of electron tunneling in the alternating electric field $P_{e}(\varepsilon)$ is calculated semiclassically using the short-radius potential model for impurities after Refs. 10 and 11. The electron wave function in an arbitrary point $\mathbf{r}$ and for

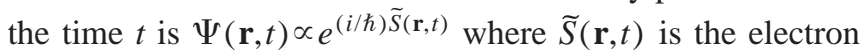
principal function (action). In order to determine $\widetilde{S}(\mathbf{r}, t)$ as a function of the electron coordinates $\mathbf{r}$ and the time $t$ one should find the general integral ${ }^{12}$ of the Hamilton-Jacobi equations

$$
\frac{\partial \widetilde{S}}{\partial t}=-\mathcal{H}(\mathbf{p}, \mathbf{r}, t), \quad \nabla \widetilde{S}=\mathbf{p},
$$

which depends on an arbitrary function. Here $\mathcal{H}$ and $\mathbf{p}$ are the Hamiltonian and the electron momentum, respectively. The resulting principal function $\widetilde{S}(\mathbf{r}, t)$ can be written in the form

$$
\widetilde{S}=\widetilde{S}_{0}-\varepsilon t_{0}, \quad \widetilde{S}_{0}=\int_{t_{0}}^{t} \mathcal{L}\left(\mathbf{r}^{\prime}, \dot{\mathbf{r}}^{\prime}, t^{\prime}\right) d t^{\prime},
$$

where $\mathcal{L}$ is the Lagrange function. The position vector $\mathbf{r}^{\prime}\left(t^{\prime}\right)$ as a function of $t^{\prime}$ can be found by solving the classical equation of motion with boundary conditions:

$$
\left.\mathbf{r}^{\prime}\left(t^{\prime}\right)\right|_{t^{\prime}=t_{0}}=0,\left.\quad \mathbf{r}^{\prime}\left(t^{\prime}\right)\right|_{t^{\prime}=t}=\mathbf{r}
$$

The principal function is obtained in the form of Eq. (6) by taking into account that at $t=t_{0}, r=0$ [Eq. (7)], and that the electron wave function $\Psi(\mathbf{r}, t)$ is equal to the unperturbed wave function $\Psi_{0}(\mathbf{r}, t)$, which is in a short-range potential at $r=0$ (at the defect) given by $\Psi_{0}(\mathbf{r}, t) \propto \exp (-i \varepsilon t / \hbar)$ with 
$\varepsilon<0$. In Eqs. (6) and (7), $t_{0}$ is a function of $\mathbf{r}$ and $t$ which should be found from the equation

$$
\left(\frac{\partial \widetilde{S}}{\partial t_{0}}\right)_{\mathbf{r}, t}=0
$$

We want to emphasize that $\mathbf{r}$ and $t$ are real while $\mathbf{r}^{\prime}, t^{\prime}$, and $t_{0}$ can be complex.

The electron tunneling probability $P_{e}(\varepsilon)$, being determined by the current density flowing from the center, is proportional to $|\Psi|^{2}$. To find $P_{e}(\varepsilon)$ it is sufficient to calculate $\operatorname{Im} \widetilde{S}$ in the vicinity of its maximum, i.e., at values of $\mathbf{r}$, where

$$
\operatorname{Im} \nabla \widetilde{S}=\operatorname{Im}\left(\left.\mathbf{p}\right|_{t^{\prime}=t}\right)=0 .
$$

For this region of space it follows that after the left-hand side of Eqs. (5),

$$
\frac{\partial(\operatorname{Im} \widetilde{S})}{\partial t}=0 .
$$

Thus, the probability of electron tunneling $P_{e}(\varepsilon)$ can be written as

$$
P_{e}(\varepsilon)=\exp \left[-2 S_{e}(\varepsilon)\right]
$$

where

$$
S_{e}(\varepsilon)=\frac{\operatorname{Im} \widetilde{S}}{\hbar}
$$

Here $\widetilde{S}$ is determined by Eqs. (6), (8), and (9). Note that in calculating $S_{e}(\varepsilon)$ one can arbitrarily take a value of the time $t$ according to Eq. (10); we will assume $t=0$.

As a result the probability of ionization $e(E)$ as a function of the electric field $E$ is obtained by integrating Eq. (1) over $\mathcal{E}$ and $\varepsilon$ :

$$
e(E)=\iint P_{e}(\varepsilon) P_{d}(\mathcal{E}) \exp \left(-\mathcal{E} / k_{B} T\right) d \varepsilon d \mathcal{E} .
$$

Calculating this integral by the saddle point method shows that there is a vibrational energy $\mathcal{E}=\mathcal{E}_{m}$ and an electron energy $\varepsilon=\varepsilon_{m}$ where the ionization probability has a sharp maximum. Thus, defect and electron tunneling take place mostly at these energy levels and the ionization probability can be written in the following approximate form:

$$
e(E) \propto P_{e}\left(\varepsilon_{m}\right) P_{d}\left(\mathcal{E}_{m}\right) \exp \left(-\mathcal{E}_{m} / k_{B} T\right) .
$$

The defect and electron tunneling at the energy levels $\mathcal{E}_{m}$ and $\varepsilon_{m}$ can be characterized by a defect tunneling time $\tau$ and an electron tunneling time $\tau_{e}(\varepsilon)$, respectively. The saddle point method applied to Eq. (13) yields that the defect tunneling time is determined by the temperature: ${ }^{4}$

$$
\tau=\tau_{2 \varepsilon}\left(\mathcal{E}_{m}, \varepsilon_{m}\right)-\tau_{1 \varepsilon}\left(\mathcal{E}_{m}, \varepsilon_{m}\right)=\frac{\hbar}{2 k_{B} T},
$$

where $\tau_{n \varepsilon}$ are tunneling times under the barriers $U_{n \varepsilon}$ of the vibrational system with

$$
\tau_{n \varepsilon}(\mathcal{E}, \varepsilon)=-\hbar \frac{\partial S_{n \varepsilon}}{\partial \mathcal{E}}, \quad n=1,2
$$

The electron tunneling time $\tau_{e}(\varepsilon)$ is determined as

$$
\tau_{e}(\varepsilon)=-\hbar \frac{\partial S_{e}}{\partial \varepsilon} .
$$

As an important result obtained by the saddle point method to solve the integral Eq. (13) is that the electron tunneling time $\tau_{e}\left(\varepsilon_{m}\right)$ is equal to the defect tunneling time $\tau_{2 \varepsilon}\left(\mathcal{E}_{m}, \varepsilon_{m}\right)$ under the potential $U_{2 \varepsilon}$ of the ionized configuration:

$$
\tau_{e}\left(\varepsilon_{m}\right)=\tau_{2 \varepsilon}\left(\mathcal{E}_{m}, \varepsilon_{m}\right)
$$

The solution of Eqs. (15) and (18) allows one to find $\mathcal{E}_{m}$ and $\varepsilon_{m}$. Using Eqs. (6) and (12) we obtain

$$
\tau_{e}(\varepsilon)=-\operatorname{Im} \frac{\partial \widetilde{S}}{\partial \varepsilon}=-\operatorname{Im}\left(-t_{0}+\frac{\partial \widetilde{S}}{\partial t_{0}} \frac{\partial t_{0}}{\partial \varepsilon}\right) .
$$

According to Eq. (8) we finally get

$$
\tau_{e}(\varepsilon)=\operatorname{Im} t_{0}
$$

\section{B. Phonon-assisted tunneling}

The tunneling ionization probability in the limit of not too high electric fields and not too low temperatures is dominated by phonon-assisted tunneling. The electric field and temperature limits will be defined more precisely below. The theory is developed for neutral impurities, which means that the Coulomb force between the carrier and center is ignored when the carrier is detached from the impurity center. The tunneling ionization of charged impurities in a static field is considered in Ref. 13, showing that at low electric fields ionization is caused by the Poole-Frenkel effect whereas at high fields tunneling ionization enhanced by the Coulomb force dominates the emission process. In the case of phononassisted tunneling the optimum electron tunneling energy $\varepsilon_{m}$ is small in comparison to the optimum defect tunneling energy $\mathcal{E}_{m}$. In this limit the tunneling emission probability $e(E)$ of carriers can be calculated analytically. The effect of the electric field is a small shift of the ionized potential $U_{2}(x)$ to a lower level $U_{2 \varepsilon}(x)$. The potential $U_{1}(x)$ is not affected by the electric field. For small $\varepsilon$ the quantities $S_{1 \varepsilon}$ and $S_{2 \varepsilon}$ can be taken into account in a linear approximation as a function of $\varepsilon$. Then we obtain $S_{2 \varepsilon}-S_{1 \varepsilon}=S_{2}-S_{1}$ $+\tau_{2} \varepsilon / \hbar$, where $S_{1}, S_{2}$, and $\tau_{2}$ are calculated after Eqs. (3), (4), and (16) for $\varepsilon=0$ and are independent of the electric field. Taking into account Eqs. (15)-(17) and (20) we find the dependence of the ionization probability on the electric field in the form

$$
e(E)=e(0) \exp \left(-\frac{2}{\hbar} \operatorname{Im} \widetilde{S}_{0}\left(\varepsilon_{m}\right)\right),
$$

where $e(0)$ is the thermal ionization probability at zero field, and $\widetilde{S}_{0}$ follows from Eq. (6) and should be calculated in the range where Eq. (9) is satisfied at arbitrary $t$. 


\section{Frequency dependence}

Now we consider the frequency dependence of phononassisted tunneling and determine $\operatorname{Im} \widetilde{S}_{0}\left(\varepsilon_{m}\right)$. If an alternating electric field $\mathbf{E}(t)$ is applied to an electron, the Lagrange function has the form

$$
\mathcal{L}\left(\mathbf{r}^{\prime}, \dot{\mathbf{r}}^{\prime}, t^{\prime}\right)=\frac{m \dot{\mathbf{r}}^{\prime 2}}{2}+e\left[\mathbf{r}^{\prime} \cdot \mathbf{E}\left(t^{\prime}\right)\right]
$$

where $e$ is electron charge. Integrating Eq. (6) by parts and taking into account Eqs. (22) and the equation of motion $m \ddot{\mathbf{r}^{\prime}}=e \mathbf{E}\left(t^{\prime}\right)$ with the boundary conditions given by Eqs. (7) and (9) we get

$$
\operatorname{Im} \widetilde{S}_{0}=-\operatorname{Im} \int_{t_{0}}^{t} \frac{m \dot{\mathbf{r}}^{\prime 2}}{2} d t^{\prime}
$$

In the following we consider the general case of elliptically polarized radiation of frequency $\omega$ propagating in the $z$ direction $\left(E_{x}=E_{1} \cos \omega t, E_{y}=E_{2} \sin \omega t\right)$.

After some calculations we get a relation between the tunneling time $\tau_{e}$ and the electron energy $\varepsilon$

$$
\begin{aligned}
\frac{2 \varepsilon}{m}= & -\left(\frac{e E_{1}}{m \omega}\right)^{2} \sinh ^{2}\left[\omega \tau_{e}(\varepsilon)\right] \\
& +\left(\frac{e E_{2}}{m \omega}\right)^{2}\left(\frac{\sinh \omega \tau_{e}(\varepsilon)}{\omega \tau_{e}(\varepsilon)}-\cosh \omega \tau_{e}(\varepsilon)\right)^{2} .
\end{aligned}
$$

Then after integration of Eq. (23) we obtain $\operatorname{Im} \widetilde{S}_{0}$ as

$$
\begin{aligned}
\operatorname{Im} \widetilde{S}_{0}= & \frac{e^{2} \tau_{e}(\varepsilon)}{4 m \omega^{2}}\left[E_{1}^{2}\left(1-\frac{\sinh 2 \omega \tau_{e}(\varepsilon)}{2 \omega \tau_{e}(\varepsilon)}\right)\right. \\
& \left.+E_{2}^{2}\left(1+\frac{\sinh 2 \omega \tau_{e}(\varepsilon)}{2 \omega \tau_{e}(\varepsilon)}-2 \frac{\sinh ^{2} \omega \tau_{e}(\varepsilon)}{\left[\omega \tau_{e}(\varepsilon)\right]^{2}}\right)\right] .
\end{aligned}
$$

In the case of phonon-assisted tunneling considered here $\left(\left|\varepsilon_{m}\right| \ll \mathcal{E}_{m}\right)$, the electron tunneling time $\tau_{e}\left(\varepsilon_{m}\right)$ is equal to the defect tunneling time $\tau_{2}$. Therefore, the probability of phonon-assisted tunneling can be obtained by using Eqs. (21) and (25) substituting $\tau_{e}\left(\varepsilon_{m}\right)$ by $\tau_{2}$. From Eq. (21) we get the ionization probability as a function of the electric field $E$ :

$$
e(E)=e(0) \exp \left(\frac{E^{2}}{E_{c}^{2}}\right) .
$$

It is convenient to write $E_{c}$ in the form

$$
E_{c}^{2}=\frac{3 m \hbar}{e^{2}\left(\tau_{2}^{*}\right)^{3}}
$$

introducing an effective time $\tau_{2}^{*}$. This time may be obtained from Eq. (25). In the case of linear polarization $\left(E_{1}=E, E_{2}\right.$ $=0)$ the effective time is given by ${ }^{1}$

$$
\left(\tau_{2}^{*}\right)^{3}=\frac{3 \tau_{2}}{2 \omega^{2}}\left(\frac{\sinh 2 \omega \tau_{2}}{2 \omega \tau_{2}}-1\right),
$$

and for circular polarization $\left(E_{1}=E_{2}=E\right)$ we have

$$
\left(\tau_{2}^{*}\right)^{3}=\frac{3 \tau_{2}}{\omega^{2}}\left(\frac{\sinh ^{2} \omega \tau_{2}}{\left(\omega \tau_{2}\right)^{2}}-1\right) .
$$

These relations show that for a static electric field $(\omega$ $=0)$ the effective time $\tau_{2}^{*}$ is equal to the defect tunneling time $\tau_{2}$. Therefore Eqs. (26) and (27) are in agreement with previous derivations of the tunneling emission probability in static fields. ${ }^{14}$

Equations (26)-(29) have been obtained without any assumption about the shape of the adiabatic potentials $U_{1}(x)$ and $U_{2}(x)$. In fact, the defect tunneling time $\tau=\hbar / 2 k_{B} T$ is independent of the form of the potentials. However, the parameters $\tau_{1}$ and $\tau_{2}=\tau+\tau_{1}$, which are crucial for electricfield-stimulated tunneling, substantially depend on the configuration of the potentials. In the Huang-Rhys model of parabolic potentials the tunneling time under potential $U_{1}(x)$ calculated after Eq. (16) for $\varepsilon=0$ is given by

$$
\tau_{1}=\frac{1}{2 \omega_{v i b}} \ln \frac{\varepsilon_{T}}{\Delta \varepsilon},
$$

where $\Delta \varepsilon=\varepsilon_{o p t}-\varepsilon_{T}$ and $\varepsilon_{o p t}$ is the optical ionization energy taking into account the Franck-Condon principle and $\omega_{v i b}$ is the local vibrational frequency. ${ }^{7}$ The tunneling time $\tau_{1}$ is negative for autolocalized impurities with $\Delta \varepsilon>\varepsilon_{T}$ and positive for substitutional impurities with weak electronphonon interaction where $\Delta \varepsilon<\varepsilon_{T}$. Thus the tunneling time under the potential $U_{2}(x)$ is given by ${ }^{4}$

$$
\tau_{2}=\frac{\hbar}{2 k T} \pm\left|\tau_{1}\right|,
$$

with the plus and minus signs for substitutional and autolocalized impurities, respectively.

The tunneling time $\tau_{2}$ controls defect tunneling in static fields, being a function of the temperature, and the shape of the adiabatic potentials is independent of the frequency $\omega$. The effective time $\tau_{2}^{*}$ which controls tunneling for all frequencies additionally depends on $\omega$. The frequency dependence of $\tau_{2}^{*} / \tau_{2}$ as function of $\omega \tau_{2}$ is displayed in Fig. 3 for linear and circular polarized radiation. As long as $\omega \tau_{2} \leqslant 1$ the ratio $\tau_{2}^{*} / \tau_{2}$ is equal to 1 and in this quasistatic regime the ionization probability is independent of the electric field frequency and the state of polarization. For $\omega \tau_{2}>1$ the ratio increases enhancing drastically the ionization probability. In this high-frequency regime the ionization probability is polarization dependent, being higher for linear compared to circular polarized radiation at the same amplitude $E$ of the electric field.

\section{Magnetic field dependence}

An external magnetic field $B$ applied perpendicularly to the electric field which generates the tunneling barrier de- 


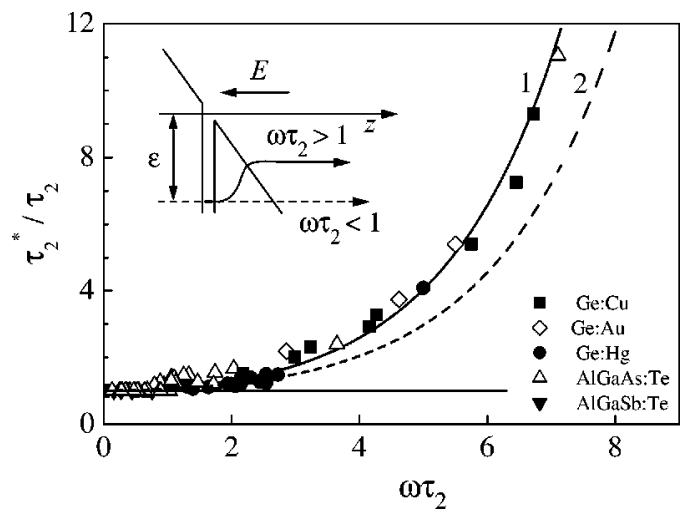

FIG. 3. Ratio $\tau_{2}^{*} / \tau_{2}$ as a function of $\omega \tau_{2}$. The curves show the dependences calculated according to Eq. (28) for linear polarization (curve 1) and Eq. (29) for circular polarization (curve 2). Experimental results obtained with linearly polarized radiation are plotted for all materials, all temperatures, and all radiation frequencies of the present investigation. Inset shows the electron tunneling trajectory in the quasistatic limit (dashed line) and the high-frequency regime (solid line).

creases the probability of electron tunneling. This effect was theoretically investigated for tunneling of electrons through static potential barriers. ${ }^{11,15}$ The theory has been extended for phonon-assisted tunneling ionization of deep impurities in dc electric fields ${ }^{16}$ and in high-frequency alternating fields ${ }^{17}$ showing that also in the case of phonon-assisted tunneling the carrier emission is suppressed by an external magnetic field. For the ionization probability we get again an exponential dependence on the square of the electric field strength $\propto \exp \left(E^{2} / E_{c}^{2}\right)$ where we write $E_{c}^{2}$ in the form of Eq. (27) defining by this an effective time $\tau_{2}^{*}$ which depends now on the magnetic field strength:

$$
\begin{aligned}
\left(\tau_{2}^{*}\right)^{3}= & \frac{3 \omega_{c}^{2}}{\left(\omega^{2}-\omega_{c}^{2}\right)^{2}} \\
& \times\left\{\int _ { 0 } ^ { \tau _ { 2 } } \left[\left(-\cosh \omega \tau+\frac{\omega_{c}}{\omega} \frac{\sinh \omega \tau_{2}}{\sinh \omega_{c} \tau_{2}} \cosh \omega_{c} \tau\right)^{2} d \tau\right.\right. \\
& \left.\left.+\int_{0}^{\tau_{2}}\left(\frac{\omega}{\omega_{c}} \sinh \omega \tau-\frac{\omega_{c}}{\omega} \frac{\sinh \omega \tau_{2}}{\sinh \omega_{c} \tau_{2}} \sinh \omega_{c} \tau\right)^{2}\right] d \tau\right\}
\end{aligned}
$$

Here $\omega_{c}=e B / m^{*}$ is the cyclotron frequency. In order to display this effect as a function of radiation frequency, $\tau_{2}^{*}$ normalized by the frequency-independent tunneling time $\tau_{2}$ is shown in Fig. 4 as a function of $\omega \tau_{2}$ for different parameters $\omega_{c} \tau_{2} \propto B$. The suppression of the tunneling probability occurs in both frequency ranges, at low frequencies when tunneling is independent of frequency as well as at high frequencies when the tunneling probability increases drastically with rising frequency. The effect of a magnetic field on tunneling is strongest if $B$ is oriented normal to the tunneling trajectory and vanishes if the $B$ is parallel to electric field.

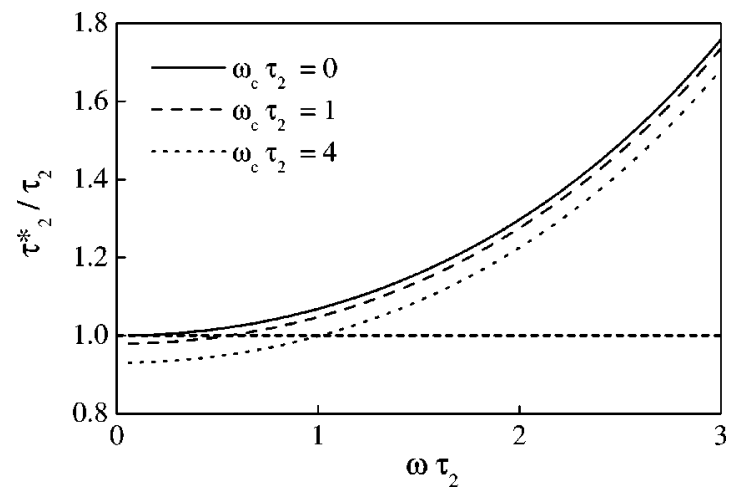

FIG. 4. The ratio $\tau_{2}^{*} / \tau_{2}$ vs $\omega \tau_{2}$ calculated after Eq. (31) for various values of $\omega_{c} \tau_{2}$.

\section{Direct tunneling ionization}

The phonon-assisted tunneling regime with the characteristic electric field dependence of ionization probability $\left[e(E) \propto \exp \left(E^{2} / E_{c}^{2}\right)\right]$ is limited by the condition that the optimal electron tunneling energy is smaller than the optimal defect tunneling energy $\left|\varepsilon_{m}\right|<\mathcal{E}_{m}$. For linear polarization $\left(E_{1}=E\right.$ and $\left.E_{2}=0\right)$, this inequality can be written in the form

$$
\frac{\left(e E \tau_{e}\right)^{2}}{2 m}<\varepsilon_{T} \frac{\left[\omega \tau_{e}(\varepsilon)\right]^{2}}{\sinh ^{2}\left[\omega \tau_{e}(\varepsilon)\right]} .
$$

If this inequality is violated, direct tunneling without involving thermal phonons dominates the ionization process. This occurs at high electric fields which increases $\left|\varepsilon_{m}\right|$. As Eq. (32) shows, the electric field strength where the transition from phonon-assisted tunneling to direct tunneling occurs decreases with increasing of $\tau_{2}$ by decreasing temperature and increasing frequency. At electric field strength $E$ $>\sqrt{2 m \varepsilon_{\text {opt }}} \omega / e$ from Eq. (32) follows that $\omega \tau_{e}(\varepsilon)<1$. In this case $\tau_{e}(\varepsilon)$ does not depend on $\omega$. In the regime of direct tunneling the ionization probability approaches the wellknown relation for electron tunneling through a triangular barrier. ${ }^{10}$ The effect of thermal phonons can be considered as a small perturbation which decays with rising electric field strength. The emission probability is found to be independent of frequency and can be written as

$$
e(E)=\frac{e E}{2 \sqrt{2 m \varepsilon_{o p t}}} \exp (-\phi)
$$

with

$$
\phi=\frac{4 \sqrt{2 m}}{3 \hbar e E} \varepsilon_{o p t}^{3 / 2}-b \frac{m \omega_{v i b} \varepsilon_{o p t}^{2}}{\hbar e^{2} E^{2}} \operatorname{coth} \frac{\hbar \omega_{v i b}}{2 k T}
$$

Here $b$ is a constant. In the Huang-Rhys model $b$ $=4 \Delta \varepsilon / \varepsilon_{\text {opt }}$ where $\Delta \varepsilon=\varepsilon_{\text {opt }}-\varepsilon_{T}$. The first term in Eq. (34) is the exponent for electron tunneling through a triangular barrier [see Fig. 1(b)] while the second term is the temperature correction. 


\section{EXPERIMENT}

The experimental investigations presented here were carried out on autolocalized centers as well as substitutional impurities. Measurements on autolocalized impurities were performed on DX centers in $\mathrm{Al}_{0.35} \mathrm{Ga}_{0.65} \mathrm{As}$ and $\mathrm{Al}_{0.5} \mathrm{Ga}_{0.5} \mathrm{Sb}$ doped with Te. ${ }^{18}$ The doping concentration was 2 $\times 10^{18} \mathrm{~cm}^{-3}$. The measurements on substitutional impurities were performed on $p$-type germanium doped with gold, mercury, and copper, having ionization energies of $\varepsilon_{T}$ $=150 \mathrm{meV}, 90 \mathrm{meV}$, and $44 \mathrm{meV}$, respectively. The doping concentrations were between $10^{14} \mathrm{~cm}^{-3}$ and $10^{15} \mathrm{~cm}^{-3}$.

As a source of terahertz electric fields a high-power, pulsed far-infrared (FIR) molecular laser pumped by a TEA-CO $\mathrm{CO}_{2}$ laser has been used. Electric field strengths in the semiconductor samples up to about $40 \mathrm{kV} / \mathrm{cm}$ corresponding to an intensity of $\simeq 5 \mathrm{MW} / \mathrm{cm}^{2}$ have been achieved in the frequency range from $\omega=3$ to $25 \mathrm{THz}$ with $40 \mathrm{~ns}$ laser pulses. To cover this wide frequency range $\mathrm{NH}_{3}, \mathrm{CH}_{3} \mathrm{~F}$, and $\mathrm{D}_{2} \mathrm{O}$ have been used as FIR laser media, yielding strong single-line linearly polarized emissions at wavelengths $\lambda$ between 76 and $560 \mu \mathrm{m}$ corresponding to photon energies ranging from 16 to $2 \mathrm{meV}$.

The samples were cooled to temperatures where at thermal equilibrium practically all carriers are frozen out on the impurities $(T=4.2-150 \mathrm{~K}$, depending on the material). The ionization of deep impurities has been detected by the farinfrared laser radiation photoconductivity measurements. ${ }^{18}$ The ratio of conductivity under illumination, $\sigma_{i}$, and dark conductivity, $\sigma_{d}$, has been determined from peak values of photoconductive signals. ${ }^{1}$ For laser pulses shorter than the carrier capture time, as is the case here, $\sigma_{i} / \sigma_{d}$ is equal to $e(E) / e(0)$, where $e(E)$ is the emission rate as a function of the electric field strength $E$. Note that the FIR response in the case of DX centers corresponds to the detachment of electrons from the defect, yielding persistent photoconductivity (see for details Ref. 4).

Experimental results will be presented for linearly polarized radiation. The photoconductive signal due to impurity ionization rises superlinearly with the intensity of incident radiation. As has been shown, ${ }^{1-4}$ the emission of carriers at photon energies smaller than the binding energy of the impurities is caused by tunneling in the alternating electric field of radiation. At not too low temperatures and not too high frequencies the tunneling probability exponentially increases with the square of the electric field strength as $e(E)$ $\propto \exp \left(E^{2} / E_{c}^{* 2}\right)$ and is independent of frequency. This behavior, characteristic for the quasistatic regime investigated in detail in Refs. 2-4 and 13, has been observed at sufficiently high temperatures. ${ }^{19}$ In this quasistatic regime $\omega \tau_{2} \ll 1$, the electron tunnels at the momentary magnitude of the electric field in a time shorter than the period of the oscillation. Thus the electric field acts like a static field and the ionization probability does not depend on frequency. The observed exponential rise of the ionization probability with the square of electric field strength corresponds to the phonon-assisted tunneling regime which is described by Eqs. (26) and (27). The characteristic field $E_{c}^{* 2}$, which can be determined experimentally, is given by $E_{c}^{* 2}=\left(3 m^{*} \hbar\right) /\left(\tau_{2}^{* 3} e^{2}\right)$ with $\tau_{2}^{*}=\tau_{2}$ [see Eq. (27)]. The experimentally determined defect tunneling times $\tau_{2}$ are in a good agreement with Eq. (30) (for details see Ref. 18).

The frequency-independent tunneling is limited to frequencies $\omega$ with $\omega \tau_{2}<1$ [see Eq. (28)]. The fact that the tunneling time $\tau_{2}$ depends on temperature [see Eq. (30)] allows one to achieve the high-frequency regime $\omega \tau_{2}>1$ simply by cooling of samples. That is an important advantage in this case because the other opportunity to reach $\omega \tau_{2} \geqslant 1$, i.e., raising of frequency, is limited by one-photon absorption. The measurements show that in a finite electric field range for the case of $\omega \tau_{2} \geqslant 1$ the ionization probability still depends exponentially on the square of the electric field strength, $e(E) \propto \exp \left(E^{2} / E_{c}^{* 2}\right) .{ }^{1}$ The essential difference compared to the $\omega \tau_{2}<1$ situation is that the characteristic field $E_{c}^{*}$ becomes now frequency dependent. It is found that ionization is enhanced with rising frequency. This behavior has been observed for all impurities at not very low temperatures and not very high electric field strength. At higher field strengths the exponential dependence on $E^{2}$ ceases and the ionization probability rises more slowly with increasing $E$. This high-field case will be discussed below.

The experimentally determined values of $E_{c}^{*}$ for various frequencies, temperatures, and materials allow one to obtain $\tau_{2}^{*} / \tau_{2} \cdot{ }^{20}$ Figure 3 shows this ratio as a function of $\omega \tau_{2}$ in comparison to calculations after Eq. (28). The tunneling times $\tau_{2}$ were determined from frequency-independent values of $E_{c}^{*}$. The experimental results shown in Fig. 3 are grouped according to the materials. For each material the variation of the value of $\omega \tau_{2}$ has been obtained by applying different radiation frequencies in the range from $3 \mathrm{THz}$ to 25 $\mathrm{THz}$ and different temperatures between $4.2 \mathrm{~K}$ and $150 \mathrm{~K}$. Good agreement between theory and experiment is obtained. It should be pointed out that the theory does not contain any fitting parameter.

The enhancement of tunneling at frequencies higher than the inverse tunneling time has been anticipated in a number of theoretical works, ${ }^{10,21-24}$ but has been demonstrated experimentally only recently. ${ }^{1}$ In contrast to static electric fields where the electron tunnels at a fixed energy, in alternating fields the energy of the electron is not conserved during tunneling. In this case the electron can absorb energy from the field (see inset in Fig. 3) and hence leaves the impurity at an effectively narrower tunneling barrier. This leads to a sharp increase of the tunneling probability with increasing frequency. The observed enhancement of ionization probability demonstrates that an electron can indeed absorb energy below a potential barrier if the process of tunneling is induced by a high-frequency alternating electric field. The absorption of energy is controlled by the electron tunneling time $\tau_{e}$, i.e., the Büttiker-Landauer time. In the case of phonon-assisted tunneling the energy of the electron under the barrier, $\varepsilon_{m}$, follows from the condition that the electron tunneling time $\tau_{e}$ be equal to the defect tunneling time $\tau_{2}$, which is determined by the tunneling reconstruction of the defect vibration system, Eq. (30). Thus in the case of phonon-assisted tunneling, the Büttiker-Landauer time of electron tunneling can be var- 


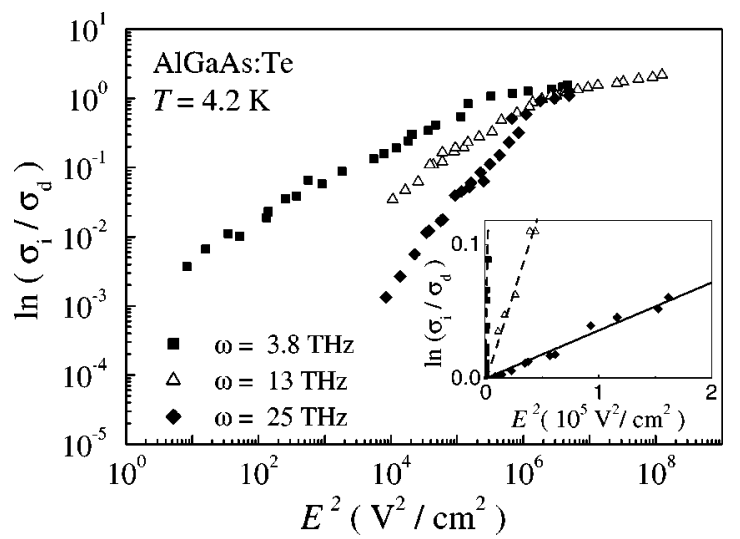

FIG. 5. $\ln \left(\sigma_{i} / \sigma_{d}\right)$ for $\mathrm{Al}_{0.35} \mathrm{Ga}_{0.65} \mathrm{As}$ :Te as a function of $E^{2}$ for different frequencies $\omega$ at liquid helium temperature. The inset shows the low-field behavior.

ied by the temperature and can be measured by the field dependence of ionization probability.

Further decrease of the temperature increases the tunneling time and leads to a much stronger frequency dependence of the ionization probability. Figure 5 shows measurements carried out at $4.2 \mathrm{~K}$ on AlGaAs:Te. ${ }^{25}$ In order to display in one figure the total set of data covering eight orders of magnitude in the square of the electric field strength, $\ln \left(E^{2}\right)$ has been plotted on the abscissa. To make an easy comparison to the $\exp \left(E^{2} / E_{c}^{2}\right)$ dependence of $\sigma_{i} / \sigma_{d}$ possible, a log-log presentation has been used for the ordinate. In the low-field range the characteristic $\propto \exp \left(E^{2} / E_{c}^{* 2}\right)$ field dependence of phonon-assisted tunneling is observed. This is shown in addition in the inset of Fig. 5 in a log-ln plot. The frequency dependence in the field range of phonon assisted tunneling is so strong that a change of three orders of magnitude of $E^{2}$ needs only a sixfold change in frequency $\omega$. Similar results have been obtained for substitutional impurities having a smaller binding energy and showing larger tunneling times $\tau_{2}$ (see Ref. 18). Figure 6 shows experimental results for $\mathrm{Ge}: \mathrm{Cu}$ at $T=4.2 \mathrm{~K}$ in the frequency range between 3.4 and $25 \mathrm{THz}$. Here the frequency dependence at low field

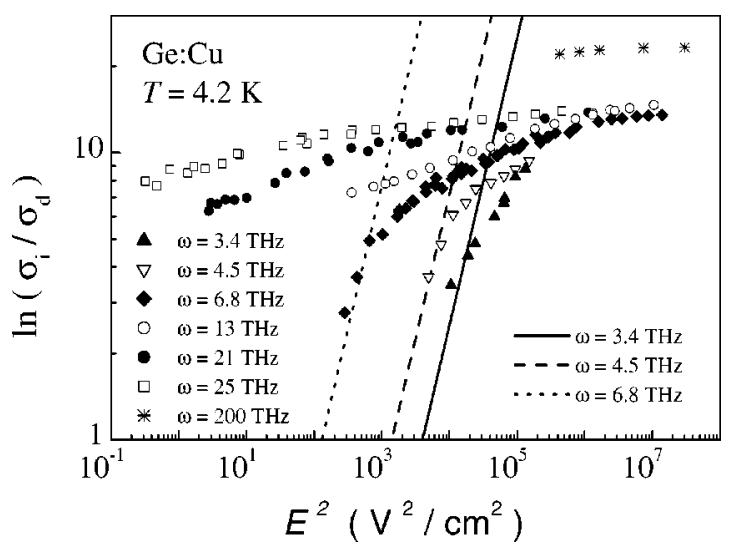

FIG. 6. $\ln \left(\sigma_{i} / \sigma_{d}\right)$ for $\mathrm{Ge}: \mathrm{Cu}$ as a function of $E^{2}$ for different frequencies $\omega$ at liquid helium temperature. Lines show calculations after Eqs. (26)-(28) for the three lowest frequencies used in experiment.

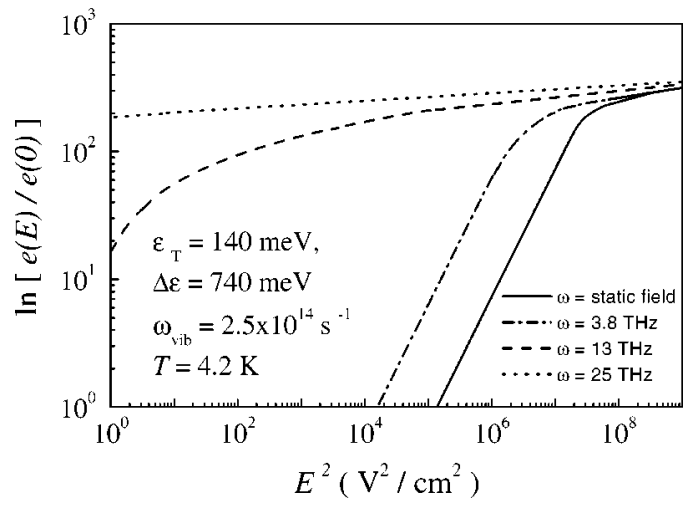

FIG. 7. Logarithm of the normalized ionization probability vs $E^{2}$ calculated for different frequencies used in experiment. Calculations are carried out for $4.2 \mathrm{~K}$ using the parameters of $\mathrm{Al}_{0.35} \mathrm{Ga}_{0.65} \mathrm{As}$ :Te taking into account phonon-assisted tunneling and direct tunneling.

strengths is even stronger. For a given constant signal, a change of six orders of magnitude of $E^{2}$ requires a factor of 7 change in frequency $\omega$.

At higher field strengths the field dependence of the emission probability gets much weaker and the frequency dependence practically disappears. The transition to frequencyindependent probability at higher field strength occurs at higher fields for DX centers in AlGaAs:Te than for Ge:Cu. The weak increase of the frequency-independent carrier emission at high electric fields cannot be attributed to emptying of the impurity states. This has been proved by onephoton ionization of $\mathrm{Ge}: \mathrm{Cu}$ using a $\mathrm{CO}_{2}$ laser radiation of $\omega=200 \mathrm{THz}$. The saturation level of photoconductivity where practically all impurities are ionized lies well above the far-infrared data (see Fig. 6).

This complex dependence of ionization probability on field strength and radiation frequency is a result of the transition from phonon-assisted tunneling at low field strengths to direct tunneling without phonons at high fields (see Sec. II C). The emission probability for phonon-assisted tunneling as a function of the electric field strength given by Eq. (26) was obtained in the limit that corrections to thermal emission resulting from electron tunneling are small; i.e., the energy of electron tunneling $\left|\varepsilon_{m}\right|$ is much smaller than the defect tunneling energy $\mathcal{E}_{m}$. In the opposite limit $\left|\varepsilon_{m}\right| \geqslant \mathcal{E}_{m}$, direct carrier tunneling from the ground state into the continuum, without participation of phonons, becomes dominant. ${ }^{3}$ Direct electron tunneling occurs at the crossing of the $U_{2 \varepsilon}(x)$ and $U_{1}(x)$ potential curves, where an electronic transition is possible without any change in the configuration coordinate. This effect, leading to a weaker field dependence of the ionization probability in comparison to that of phonon-assisted tunneling, dominates the ionization process at very high fields.

Figure 7 shows the result of the calculations using parameters of DX centers in AlGaAs:Te. The calculations, which take into account both phonon-assisted tunneling and direct tunneling processes, are carried out for several field frequencies used in the experiments. The calculations are performed for the Huang-Rhys adiabatic potential model. ${ }^{7}$ The prob- 


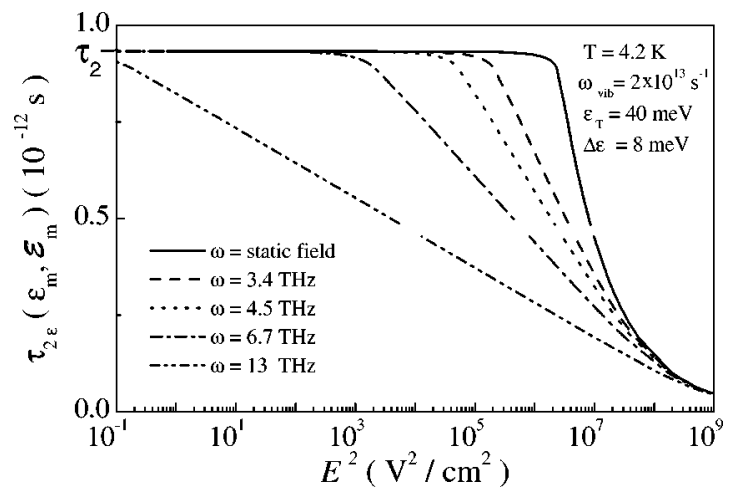

FIG. 8. The tunneling time $\tau_{2 \varepsilon}\left(\varepsilon_{m}, \mathcal{E}_{m}\right)$ vs $E^{2}$ calculated for different frequencies used in experiment. Calculation are carried out for $4.2 \mathrm{~K}$ using the parameters of $\mathrm{Ge}: \mathrm{Cu}$ taking into account phonon-assisted tunneling and direct tunneling but ignoring Coulomb interactions.

ability of tunneling ionization has been calculated by using Eq. (14). For the calculations the defect tunneling times, and the electron tunneling time, and the values of optimal defect and electron tunneling energies are needed. The defect tunneling times $\tau_{2 \varepsilon}$ and $\tau_{1 \varepsilon}$ as a function of the defect energy $\mathcal{E}$ and the electron energy $\varepsilon$ have been calculated after Eq. (16). The electron tunneling time as a function of electron energy $\varepsilon$, electric field strength $E$, and radiation frequency $\omega$ has been obtained using Eq. (24) for $E_{2}=0$ and $E_{1}=E$ which corresponds to linearly polarized radiation. Optimal electron and defect tunneling energies $\varepsilon_{m}$ and $\mathcal{E}$ have been obtained using Eqs. (15) and (18).

The theory qualitatively describes all the features of the tunneling ionization probability as a function of frequency and electric field strength. The experimentally observed weaker frequency dependence of the ionization probability of AlGaAs:Te compared to $\mathrm{Ge}: \mathrm{Cu}$ is caused by the smaller values of $\tau_{2}$ in the first case. The disappearance of the frequency dependence at very high fields is caused by the reduction of the tunneling time $\tau_{2 \varepsilon}$ with rising electric field strength. In Fig. 8 the electric field dependence of $\tau_{2 \varepsilon}$ is shown, calculated with the parameters for $\mathrm{Ge}: \mathrm{Cu}$ for $4.2 \mathrm{~K}$ and various electric field frequencies. The physical reason of the drop of $\tau_{2 \varepsilon}$ is the increase of the electron tunneling en- $\operatorname{ergy} \varepsilon$. Due to this, $\omega \tau_{2}$ becomes smaller than 1 and thus the frequency dependence vanishes.

The fact that the exponential dependence on the square of the electric field strength changes to a weaker field dependence at higher fields for AlGaAs:Te compared to $\mathrm{Ge}: \mathrm{Cu}$ is caused by the difference of the binding energies. The transition from phonon-assisted tunneling to direct tunneling depends substantially on the value of the binding energy, Eq. (33). For smaller binding energies it occurs at lower fields, yielding weaker field and frequency dependences.

\section{SUMMARY}

In summary, tunneling ionization of deep impurities in semiconductors has been investigated in theory and experiment. In contrast to tunneling ionization of atoms achieved by applying very short high power pulses of visible lasers, tunneling ionization of atomlike centers in solids strongly depends on the electron-phonon interaction with the thermal bath over a wide range of electric field strength. Only at very high electric fields does tunneling occur as in the case of atoms directly without involving phonons. Due to the reconstruction of the vibrational system by tunneling detachment of a carrier, the electron-phonon interaction determines the tunneling time. Hence the tunneling time can easily be varied by changing the temperature.

The observed enhancement of the tunneling probability by increasing the frequency gives evidence that the tunneling carrier can absorb energy during tunneling under the barrier. The enhancement of tunneling occurs when the frequency becomes larger than the inverse tunneling time. This limit is approached in the terahertz regime where high electric fields can easily be applied in a contactless manner by powerful far-infrared lasers. Applying a magnetic field normal to the electric field vector, an increase of temperature and/or an increase of electric field leads to a shift of the boundary between the quasistatic and frequency-dependent regime to higher frequencies.

\section{ACKNOWLEDGMENTS}

We thank Elke Ziemann for helpful discussions. Financial support by the DFG and the RFFI is gratefully acknowledged.
${ }^{1}$ S.D. Ganichev, E. Ziemann, Th. Gleim, W. Prettl, I.N. Yassievich, V.I. Perel, I. Wilke, and E.E. Haller, Phys. Rev. Lett. 80, 2409 (1998).

${ }^{2}$ S.D. Ganichev, W. Prettl, and P.G. Huggard, Phys. Rev. Lett. 71, 3882 (1993).

${ }^{3}$ S.D. Ganichev, I.N. Yassievich, and W. Prettl, Semicond. Sci. Technol. 11, 679 (1996).

${ }^{4}$ S.D. Ganichev, J. Diener, I.N. Yassievich, W. Prettl, B.K. Meyer, and K.W. Benz, Phys. Rev. Lett. 75, 1590 (1995).

${ }^{5}$ M. Büttiker and R. Landauer, Phys. Rev. Lett. 49, 1739 (1982).

${ }^{6}$ R. Landauer and Th. Martin, Rev. Mod. Phys. 66, 217 (1994).

${ }^{7}$ V. N. Abakumov, V. I. Perel, and I. N. Yassievich, in Nonradiative
Recombination in Semiconductors, edited by V. M. Agranovich and A. A. Maradudin, Modern Problems in Condensed Matter Sciences Vol. 33 (North-Holland, Amsterdam, 1991).

${ }^{8}$ P. T. Landsberg, Recombination in Semiconductors (Cambridge University Press, New York, 1991).

${ }^{9}$ T. Markvart, J. Phys. C 17, 6303 (1984).

${ }^{10}$ L. D. Landau, and E. M. Livshitz, Quantum Mechanics (Pergamon, Oxford, 1977), pp. 295-297.

${ }^{11}$ V.S. Popov, and A.V. Sergeev, Zh. Éksp. Teor. Fiz. 113, 2047 (1998) [JETP 86, 1122 (1998)].

${ }^{12}$ L. D. Landau, and E. M. Livshitz, Mechanics (Pergamon, Oxford, 1969), pp. 138-149. 
${ }^{13}$ S.D. Ganichev, E. Ziemann, I.N. Yassievich, W. Prettl, A. Istratov, and E.R. Weber, Phys. Rev. B 61, 10361 (2000).

${ }^{14}$ V. Karpus, and V.I. Perel, Zh. Éksp. Teor. Fiz. 91, 2319 (1986) [Sov. Phys. JETP 64, 1376 (1986)].

${ }^{15}$ L.P. Kotova, A.M. Perelomov, and V.S. Popov, Zh. Éksp. Teor. Fiz. 54, 1151 (1968) [Sov. Phys. JETP 27, 616 (1968)].

${ }^{16}$ V.I. Perel and I.N. Yassievich, Pis'ma Zh. Éksp. Teor. Fiz. 68, 763 (1998) [JETP Lett. 68, 804 (1998)].

${ }^{17}$ A.S. Moskalenko, S.D. Ganichev, V.I. Perel, and I.N. Yassievich, Physica B 273-274, 1007 (1999).

${ }^{18}$ E. Ziemann, S.D. Ganichev, I.N. Yassievich, V.I. Perel, and W. Prettl, J. Appl. Phys. 87, 3843 (2000).

${ }^{19}$ Note that in the case of charged impurities (all substitutional impurities investigated here) a deviation from the field dependence $e(E) \propto \exp \left(E^{2} / E_{c}^{* 2}\right)$ can be seen in relatively low field strengths (up to $1 \mathrm{kV} / \mathrm{cm}$ ) where the defects are thermally ionized through the Poole-Frenkel effect and the ionization probability is proportional to $e(E) \propto \exp \left[\sqrt{\left(Z e^{3} E / \kappa\right)} / k_{B} T\right]$ (Ref. 13).

${ }^{20}$ For the case of substitutional impurities as for quasistatic regime the impurity charge should be taken into account.
${ }^{21}$ L.V. Keldysh, Zh. Éksp. Teor. Fiz. 47, 1945 (1964) [Sov. Phys. JETP 20, 1307 (1965)].

${ }^{22}$ Yu.A. Bychkov and A.M. Dykhne, Zh. Éksp. Teor. Fiz. 58, 1734 (1970) [Sov. Phys. JETP 31, 928 (1970)].

${ }^{23}$ B.I. Ivlev and V.I. Mel'nikov, Phys. Rev. Lett. 55, 1614 (1985).

${ }^{24}$ M.V. Ammosov, N.B. Delone, and V.P. Krainov, Zh. Éksp. Teor. Fiz. 91, 2008 (1986) [Sov. Phys. JETP 64, 1191 (1986)].

${ }^{25}$ The data have been obtained making use of persistent photoconductivity of the sample in order to reduce the dark resistance. Because of the large binding energy of the DX center, the resistance of the sample cooled in the dark is too high to detect any signal at this temperature in response to far-infrared radiation. Therefore the sample was illuminated for a short time with weak visible and near-infrared light, detaching the electrons from a small fraction of DX centers. As a result the sample resistance drops to experimentally reasonable values of the order of several hundred megohms. After this illumination the resistance change at $4.2 \mathrm{~K}$ is persistent and allows one to measure the electron detachment process by far-infrared radiation. 